\title{
DISJOINTNESS AND WEAK MIXING OF MINIMAL SETS
}

\section{K. E. PETERSEN ${ }^{1}$}

In [3, Problem G, p. 34] the following problem is proposed: characterize the class $D^{\perp}$ of all flows which are disjoint from every distal flow. We show here that $D^{\perp}$ consists precisely of the weakly mixing minimal flows. In arriving at this conclusion we make use of a result (Corollary to Theorem 1 ) which has been arrived at independently by Keynes and Robertson [4, Theorem 3.4, p. 366]. In the following all transformation groups will be assumed to have compact Hausdorff phase spaces. For any unexplained notation or terminology the reader is referred to [1] and [3]. This research is part of the author's doctoral dissertation prepared at Yale University under the guidance of Professor S. Kakutani, whose assistance the author gratefully acknowledges.

We say that a transformation group $(X, T)$ is weakly mixing if given nonempty open subsets $A, B, C, D$ of $X$ there is $t \in T$ such that $A t \cap C \neq \varnothing$ and $B t \cap D \neq \varnothing$.

Lemma. Let $(X, T)$ be a transformation group and suppose that $T$ is abelian. Then $(X, T)$ is weakly mixing if and only if given nonempty open subsets $A$ and $B$ of $X$ there is $t \in T$ such that $A t \cap A \neq \varnothing$ and $A t \cap B \neq \varnothing$.

Proof. It is clear that if $(X, T)$ is weakly mixing then the stated condition is satisfied. Suppose then that the condition holds and let nonempty open subsets $A, B, C, D$ of $X$ be given; we need to find $t \in T$ such that $A t \cap C \neq \varnothing$ and $B t \cap D \neq \varnothing$.

We may choose $t_{1} \in T$ such that $E=A t_{1} \cap B \neq \varnothing, t_{2} \in T$ such that $F=E t_{2} \cap C t_{1} \neq \varnothing$, and $t_{3} \in T$ such that $F t_{3} \cap F \neq \varnothing$ and $F t_{3} \cap D \neq \varnothing$. Let $t=t_{2} t_{3}$. Then

$$
\begin{aligned}
(A t \cap C) t_{1} & =A t_{1} t \cap C t_{1} \supseteq A t_{1} t \cap B t \cap C t_{1}=\left(A t_{1} \cap B\right) t \cap C l_{1} \\
& =E\left(t_{2} t_{3}\right) \cap C t_{1} \supseteq F t_{3} \cap C t_{1} \supseteq F t_{3} \cap F \neq \varnothing,
\end{aligned}
$$

so $A t \cap C \neq \varnothing$; and

$$
A t_{1} t \cap B t \cap D=\left(A t_{1} \cap B\right) t \cap D=E\left(t_{2} t_{3}\right) \cap D \supseteq F t_{3} \cap D \neq \varnothing,
$$

so $B t \cap D \neq \varnothing$.

Received by the editors June 17, 1969.

1 This research partially supported by Army Research Office (Durham). 
If $(X, T)$ is a transformation group, we will denote by $R P$ and $S_{\theta}$ the regionally proximal and equicontinuous structure relations of $X$, respectively [1, Definition 6, p. 261 and Definition 4, p. 260].

THEOREM 1. If $(X, T)$ is a weakly mixing transformation group, then $R P=X \times X$. If $(X, T)$ is a minimal abelian transformation group for which $S_{e}=X \times X$, then $(X, T)$ is weakly mixing.

Proof. The first statement in the theorem is easily verified. Suppose then that $(X, T)$ is a minimal abelian transformation group for which $S_{e}=X \times X$. By a result of Veech $[5$, Theorem 1.1, p. 723], this implies that given $x \in X$ and an open neighborhood $U$ of $X$, the set $\left\{x t_{1} t_{2}^{-1}: x t_{1} \in U, x t_{2} \in U\right\}$ is dense in $X$. Let nonempty open subsets $A$ and $B$ of $X$ be given, and let $x \in A$. Then there are $t_{1}, t_{2} \in T$ such that $x t_{1} \in A, x t_{2} \in A$, and $x t_{1} t_{2}^{-1} \in B$. Thus $A\left(t_{1} t_{2}^{-1}\right) \cap B \neq \varnothing$. Since $x t_{2} \in A$, we have $x \in A t_{2}^{-1}$ and $x t_{1} \in A t_{2}^{-1} t_{1}=A t_{1} t_{2}^{-1}$, and hence $x t_{1} \in A\left(t_{1} t_{2}^{-1}\right) \cap A$. The lemma now implies that $(X, T)$ is weakly mixing.

Corollary. For a minimal abelian transformation group $(X, T)$, with $X$ compact metric, the following statements are equivalent:

(1) $(X, T)$ is weakly mixing.

(2) $R P=X \times X$.

(3) $S_{e}=X \times X$.

(4) $(X, T)$ has no nontrivial distal homomorphic image.

Proof. That (1), (2), and (3) are equivalent is direct from the theorem, remembering that $R P \subseteq S_{e}$. That (3) is equivalent to (4) is proved with the aid of $[2$, Theorem 2.4, p. 483].

By a flow $(X, \phi)$ we will mean a compact metric space $X$ together with a homeomorphism $\phi: X \rightarrow X$. We denote by $D$ the class of all distal flows, by $\mathfrak{T}$ the class of all minimal flows, and by $W$ the class of all weakly mixing flows. If $\mathfrak{C}$ is a class of flows, $\mathfrak{C}^{\perp}$ will denote the class of all flows which are disjoint [3, Definition II.1, p. 24] from every flow in $\mathfrak{C}$.

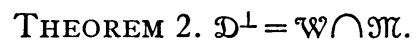

Proof. It is proved in $[3$, Theorem II.3, p. 33] that $(D \cap \Re) \perp \supseteq W$, so we have $(D \cap \Re) \perp \cap \Re \supseteq \rightsquigarrow \cap \Re$. If $(X, \phi) \in(D \cap \Re) \perp \cap \Re$, then $(X, \phi)$ can have no nontrivial distal homomorphic image, since it would not be disjoint from such an image. By the above Corollary, then, $(X, \phi) \in W \cap \Re$. Therefore $(D \cap \mathscr{T})^{\perp} \cap \mathfrak{T}=\mathscr{W} \cap \mathfrak{T}$.

It is a result of Furstenberg's [3, Theorem II.1, p. 32] that if two flows are disjoint then one of them must be minimal. Since $D$ contains 
flows which are not minimal, we have $D^{\perp} \cap \Re=D^{\perp}$. Since $D \cap \Re \subseteq \subseteq$, we have $(\mathscr{D} \cap \mathfrak{T})^{\perp} \supseteq D^{\perp}$, and hence $(\mathscr{D} \cap \mathfrak{T})^{\perp} \cap \mathfrak{M} \supseteq D^{\perp} \cap \mathfrak{M}=\mathbb{D}^{\perp}$. Thus $W \cap \Re \supseteq D^{\perp}$.

Now let $(X, \phi) \in W \cap \mathscr{T}$ and $(Y, \psi) \in \mathscr{D}$; we need to prove that $(X, \phi)$ and $(Y, \psi)$ are disjoint. This amounts to showing [3, Lemma II.1, p. 24] that if $V$ is a closed invariant subset of the flow $(X \times Y, \phi \times \psi)$ which projects onto $X$ and onto $Y$, then $V=X \times Y$. Suppose that $V$ is such a set. It is a well-known result of Ellis that, $(Y, \psi)$ being distal, $Y$ is the disjoint union of the minimal subsets $Y_{\alpha}$ of $(Y, \psi)$. Thus it suffices to prove that $V \supseteq X \times Y_{\alpha}$ for each $\alpha$. Now for each $\alpha,\left(Y_{\alpha}, \psi\right)$ $\in \mathscr{D} \cap \mathfrak{T}$, so by the first part of the proof $(X, \phi)$ and $\left(Y_{\alpha}, \psi\right)$ are disjoint; therefore $\left(X \times Y_{\alpha}, \phi \times \psi\right)$ is minimal. Since $V \cap\left(X \times Y_{\alpha}\right)$ is a nonempty closed invariant subset of $\left(X \times Y_{\alpha}, \phi \times \psi\right)$, we must have $V \cap\left(X \times Y_{\alpha}\right)=X \times Y_{\alpha}$. Therefore $V=X \times Y$ and the proof is complete.

We remark that as an application of these results it can be proved that a minimal flow which is prime in the sense that it has no proper homomorphic images must be either a translation on a cyclic group of prime order or else weakly mixing. We conjecture that the corollary to Theorem 1 remains valid if the requirement that $X$ be metrizable is dropped.

\section{REFERENCES}

1. Robert Ellis and W. H. Gottschalk, Homomorphisms of transformation groups, Trans. Amer. Math. Soc. 94 (1960), 258-271. MR 23 \#A960.

2. H. Furstenberg, The structure of distal flows, Amer. J. Math. 85 (1963), 477515. MR $28 \# 602$.

3. - Disjointness in ergodic theory, minimal sets, and a problem in Diophantine approximation, Math. Systems Theory 1 (1967), 1-49. MR 35 \#4369.

4. H. B. Keynes and J. B. Robertson, Eigenvalue theorems in topological transformation groups, Trans. Amer. Math. Soc. 139 (1969), 359-370.

5. W. A. Veech, The equicontinuous structure relation for minimal abelian transformation groups, Amer. J. Math. 90 (1968), 723-732. MR 38 \#702.

YALE UNIVERSITY AND

University of North Carolina at Chapel Hill 